Ann. Biol. anim. Bioch. Biophys., 1979, 19 (1A), 125-134.

\title{
Les cycles alimentaires des Vipères européennes dans des conditions semi-naturelles
}

\author{
par H. SAINT-GIRONS
}

\begin{abstract}
Laboratoire d'Evolution des Etres organisés, Université Pierre ef Marie Curie, 105, boulevard Raspail, 75006 Paris, France.
\end{abstract}

Summary. Feeding cycles of vipera maintained in semi natural conditions.

Regular observations, over a period of 5 consecutive years, of vipers living in large enclosures exposed to natural climatic conditions :

1) Confirm and improve the precision of earlier data obtained in the field on the length of the feeding season and variations due to species, age and sexual activity.

2) Show that in the study area, where summers are generally cool, the average annual maintenance ration is approximately 115 p. 100 of the body weight. This proportion will increase by a third during exceptionnaly hot summers, without increasing the length of the feeding season. The total amount of food ingested annually in young growing males is about 190 p. 100 of the initial body weight and 276 p. 100 in females which have just given birth and are replenishing their reserves. Reproducing females on the other hand consume only about 55 p. 100 of their body weight per year.

3) Reveal the existence of short-term feeding cycles. Each of these feeding periods may consist of no more than a single large meal but usually they last for 2-3 weeks. An animal would normally have 3-4 feeding periods per year but this may increase to 5 in post-partum females or decrease to 2 in adults in good condition, and even fall to 1 in reproducing females which do not feed during the period of gestation. Evidently these feeding periods are influenced by a multiplicity of factors and their rhythm tends to synchronize with that of moulting. The results obtained indicate nonetheless that these feeding periods follow a proper endogenous rhythm.

\section{Introduction.}

La plupart des Serpents se nourrissent en avalant, en entier, des proies volumineuses pesant souvent entre 20 et 30 p. 100 de leur propre poids. La digestion est donc longue et les repas plus ou moins espacés.

Les observations faites dans la nature suggèrent que beaucoup d'espèces ne s'alimentent que pendant une partie de leur vie active et qu'il existe, de ce point de vue, des différences liées à l'âge et à l'état sexuel (Saint-Girons, 1952 ; Prestt, 1971). Par ailleurs, les éleveurs savent depuis longtemps qu'un individu captif, pourtant soustrait aux variations climatiques saisonnières, ne se nourrit pas toujours à intervalles 
réguliers (Rollinat, 1934 ; Klauber, 1956). On manque toutefois de données précises à ce sujet et, comme les études dans le milieu naturel ne permettent pas de suivre longtemps le même animal, les cycles alimentaires des Serpents restent très mal connus. Les mêmes remarques s'appliquent à leurs besoins nutritifs. Il nous a donc semblé utile de donner les résultats d'observations portant sur un assez grand nombre de Vipères placées dans des conditions semi-naturelles.

\section{Matériel et méthodes.}

Une trentaine de Vipères adultes sont élevées depuis 1972 dans quatre enclos de plein air, de $9 \mathrm{~m}^{2}$, placés à l'orée d'un bois. Ces enclos, simplement entourés d'un grillage, sont aménagés de façon à ce que les captifs disposent d'une large gamme de micro-climats (Saint-Girons, 1978). Leur cycle annuel est d'ailleurs identique à celui de témoins libres vivant à proximité immédiate. Les observations sur les cycles alimentaires, poursuivies de 1973 à 1977, portent sur 5 espèces : Vipera berus, V. seoanei, V. aspis, $V$. latastei ef $V$. ammodyfes. Le poids des différents individus était compris entre 65 et $292 \mathrm{~g}$, selon l'âge et l'espèce.

Il n'a été tenu compte que des observations faites sur des sujets habitués à la captivité et en très bon état. En raison de cette sélection sévère, le travail ne porte que sur 108 année-individus, dont 9 proviennent de jeunes mâles récemment matures et encore en pleine croissance, 44 de grands mâles adultes et 55 de grandes femelles adultes.

La nourriture des Vipères consiste essentiellement en petits Rongeurs (Campagnols, Mulots ou Souris), d'un poids variant de 15 à $30 \mathrm{~g}$. Toutefois, les petits Serpents reçoivent, dans la mesure du possible, des proies plus petites, jeunes des espèces précédentes ou Soricidés, tandis que les grands spécimens disposent parfois de jeunes Rats ou de Lérots de plus de $30 \mathrm{~g}$. Les proies sont données mortes, après avoir été pesées. La nourriture est distribuée au moins deux fois par semaine, en général tous les deux jours, parfois tous les jours lors des périodes d'alimentation intense, et en léger excès, de façon à ce qu'aucun individu ne subisse un jeûne forcé de plus de 2 ou 3 jours au maximum.

Le cycle annuel d'activité des Vipères, tel qu'il se présente dans l'ouest de la France, a déjà fait l'objet de plusieurs publications (Saint-Girons, 1952, 1957, 1976 ; Duguy, 1963) et peut être brièvement résumé. Chez toutes les espèces, les premières sorties de printemps ont lieu au cours de la première quinzaine de mars chez les mâles, deux ou trois semaines plus tard chez les femelles. L'hivernage commence généralement au début de novembre.

En ce qui concerne le cycle sexuel des mâles, les espèces étudiées ici se répartissent en deux groupes. Chez Vipera berus et $V$. ammodytes, la spermatocytogenèse a lieu à la fin de l'été et au début de l'automne et la spermiogenèse au printemps. II y a une seule période d'accouplement, au printemps, qui ne commence qu'après une première mue prénuptiale ef précoce. Chez $V$. seoanei, $V$. aspis et $V$. latastei, la spermatocyłogenèse a lieu en été, il existe une première poussée spermiogénétique en septembre et une seconde au printemps, ainsi que deux périodes d'accouplement, l'une automnale et l'autre vernale. Ces trois espèces ont soit deux mues par an, en mai-juin et en juillet-août, soit trois mues par an, en mai, juillet et fin août ou début 
septembre. Dans tous les cas, la première mue est postnuptiale et assez tardive. $V$. berus a toujours deux mues annuelles, en avril et en juillet, alors que $V$. ammodytes mue régulièrement trois fois par an, à la fin de mars ou au début d'avril, en juillet et à la fin d'août ou au débuł de septembre.

Sous un climat aflantique, à été frais, les femelles ne se reproduisent que tous les deux ou trois ans, une année étant consacrée à la vitellogenèse et à la gestation, la ou les autres à la reconstitution des réserves. Pour un même stade du cycle sexuel, le cycle annuel d'activité des femelles présente beaucoup moins de différences interspécifiques que celui des mâles, mise à part l'absence d'accouplement automnal chez $V$. berus et $V$. ammodytes. II y a normalement deux mues par an, en juin et en coût, parfois trois mues (en mai, à la fin de juin ou au début de juillet et en août) chez les femelles qui ne sont ni reproductrices, ni postparturientes. En Loire-Atlantique, la mise bas a lieu régulièrement à la fin d'août chez $V$. berus, en septembre ou octobre chez les autres espèces, à une date variable d'un individu et d'une année à l'autre. Contrairement aux mâles, les femelles continuent à s'alimenter normalement pendant les périodes d'accouplement.

Dans le texte ef le tableau, les moyennes sont suivies de l'erreur standard. Le test $t$ de Słudent a été utilisé pour comparer les moyennes.

\section{TABLEAU 1}

Durée de la saison d'alimentation ef rations alimentaires annuelles chez les Vipères

\begin{tabular}{|c|c|c|c|c|c|c|c|c|c|c|}
\hline & \multicolumn{4}{|c|}{$\begin{array}{l}\text { Durée de la saison } \\
\text { d'alimentation (en jours) }\end{array}$} & \multirow[b]{2}{*}{$n$} & \multicolumn{5}{|c|}{$\begin{array}{c}\text { Quantité de nourriture } \\
\text { absorbée }\left({ }^{1}\right)\end{array}$} \\
\hline & $\mathrm{mn}$ & $m x$ & $\mathrm{~m}$ & $\sigma / \sqrt{n}$ & & $\mathrm{mn}$ & $\mathrm{mx}$ & m & $\sigma / \sqrt{n}$ & $\mathrm{n}$ \\
\hline \multicolumn{11}{|l|}{$\begin{array}{l}\text { Grands mâles adul- } \\
\text { tes }\end{array}$} \\
\hline $\begin{array}{l}\text { Années normales. . } \\
1976 \ldots \ldots \ldots \ldots \ldots \\
\text { Jeunes mâles matu- } \\
\text { res }\end{array}$ & $\begin{array}{l}39 \\
59\end{array}$ & $\begin{array}{r}154 \\
99\end{array}$ & $\begin{array}{l}84,29 \pm \\
78,15 \pm\end{array}$ & $\begin{array}{l}4,34 \\
3,99\end{array}$ & $\begin{array}{l}31 \\
13\end{array}$ & $\begin{array}{r}81 \\
113\end{array}$ & $\begin{array}{l}141 \\
191\end{array}$ & $\begin{array}{l}112,75 \\
142,06 \pm\end{array}$ & $\begin{array}{r}5,54 \\
10,94\end{array}$ & $\begin{array}{r}19 \\
8\end{array}$ \\
\hline $\begin{array}{l}\text { Années normales. } \\
1976 \ldots \ldots \ldots \ldots . . . . . . \\
\text { Femelles non repro- } \\
\text { ductrices ni post- } \\
\text { parturientes }\end{array}$ & $\begin{array}{l}73 \\
97\end{array}$ & $\begin{array}{l}128 \\
102\end{array}$ & $\begin{array}{c}105,00 \\
99,50\end{array}$ & 7,75 & $\begin{array}{l}7 \\
2\end{array}$ & $\begin{array}{l}169 \\
177\end{array}$ & $\begin{array}{l}222 \\
180\end{array}$ & $\begin{array}{l}189,35 \pm \\
178,50\end{array}$ & 8,02 & $\begin{array}{l}7 \\
2\end{array}$ \\
\hline $\begin{array}{l}\text { Années normales. } \\
1976 \ldots \ldots \ldots \ldots \\
\text { Femelles reproduc- } \\
\text { trices }\left({ }^{2}\right)\end{array}$ & $\begin{array}{l}68 \\
59\end{array}$ & $\begin{array}{r}161 \\
96\end{array}$ & $\begin{array}{l}96,92 \pm \\
81,50 \pm\end{array}$ & $\begin{array}{l}7,01 \\
7,90\end{array}$ & $\begin{array}{r}12 \\
4\end{array}$ & $\begin{array}{l}105 \\
163\end{array}$ & $\begin{array}{l}133 \\
171\end{array}$ & $\begin{array}{l}117,37 \pm \\
167,00\end{array}$ & 4,17 & $\begin{array}{l}6 \\
2\end{array}$ \\
\hline $\begin{array}{l}\text { Années normales. } \\
\text { Femelles postpartu- } \\
\text { rientes }\left({ }^{3}\right) \ldots \ldots \ldots\end{array}$ & 20 & 115 & $66,80 \pm$ & 8,2 & 10 & 32 & 75 & $55,40 \pm$ & 8,10 & 5 \\
\hline $\begin{array}{l}\text { Années normales. } \\
1976 \ldots \ldots \ldots \ldots\end{array}$ & $\begin{array}{l}124 \\
101\end{array}$ & $\begin{array}{l}148 \\
120\end{array}$ & $\begin{array}{l}135,50 \pm \\
111,30 \pm\end{array}$ & $\begin{array}{l}5,32 \\
5,55\end{array}$ & $\begin{array}{l}6 \\
3\end{array}$ & $\begin{array}{l}244 \\
279\end{array}$ & $\begin{array}{l}300 \\
300\end{array}$ & $\begin{array}{l}276,00 \pm \\
289,50 \pm\end{array}$ & 11,66 & $\begin{array}{l}5 \\
2\end{array}$ \\
\hline
\end{tabular}

Pour chaque résultat, nous donnons successivement le minimum, le maximum, la moyenne, l'erreur standard ef le nombre de données.

(1) En p. 100 du poids de la Vipère, pesée au début de la saison d'alimentation.

$\left({ }^{2}\right)$ Vipera berus non comprise. Voir le texte pour cette espèce, ainsi que pour l'année 1976.

(3) A l'exception des femelles ayant un cycle sexuel annuel ou biennal. 


\section{Résultats.}

\section{Mâles}

Durée de la saison d'alimentation et quantité de nourriture ingérée. - La durée totale de la saison d'alimentation - soit le nombre de jours qui sépare le premier repas du dernier - est en moyenne de 84 jours, mais varie beaucoup d'un individu à l'autre (tabl. 1), sans qu'il y ait de différences significatives entre les espèces. Pour des Vipères de même taille, le nombre des repas au cours de la saison d'alimentation varie moins que la durée de celle-ci et il n'y a d'ailleurs pas de liaison entre nombre des repas et durée de la saison d'alimentation.

La quantiłé relative de nourriture ingérée annuellement est faible, en moyenne 113 p. 100 du poids de la Vipère, les différences interspécifiques étant faibles et non

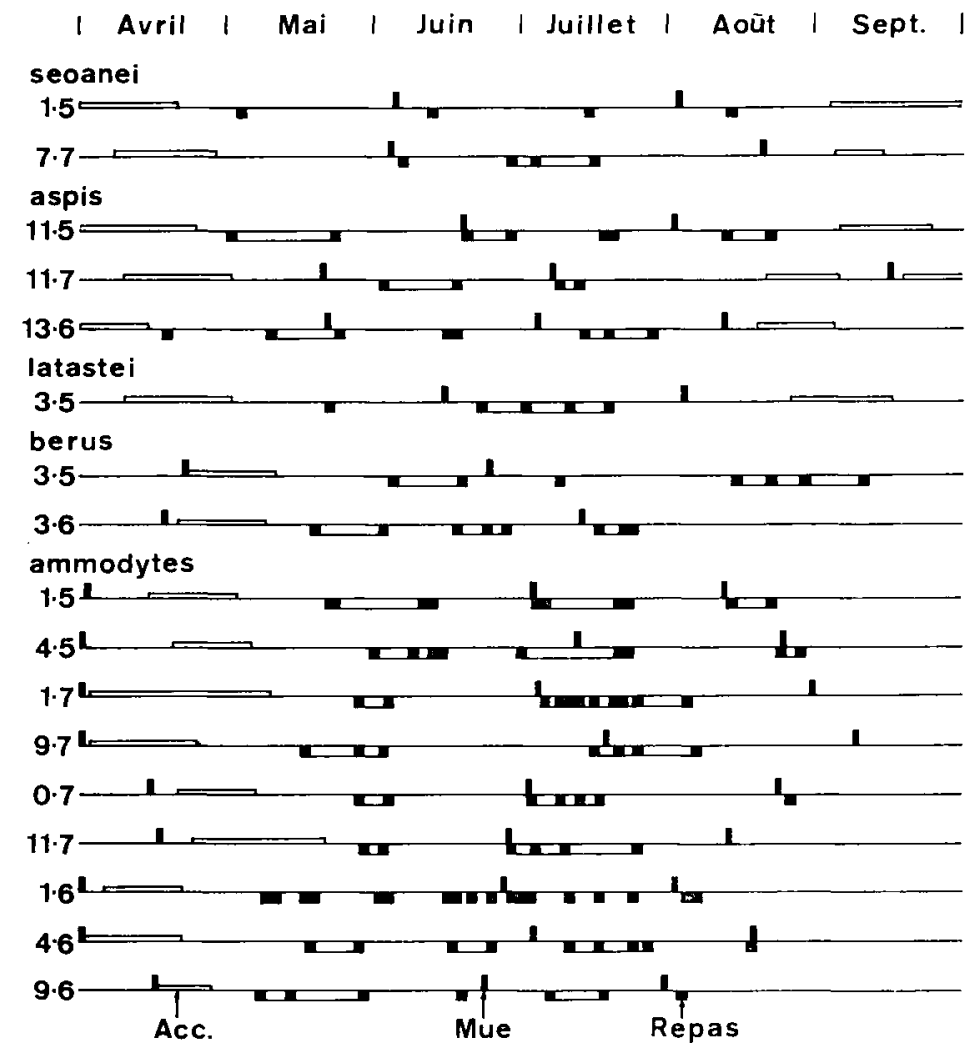

FIG. 1. - Cycles alimentaires, mues ef périodes d'accouplement chez quelques Vipères mâles (résultats individuels). Le premier chiffre, à gauche, indique le no de l'animal, le deuxième chiffre l'année des observations, en abrégé (1.5 = Vipère $n^{\circ} 1$, au cours de l'année 1975). Acc. = période d'accouplement. Chez Vipera ammodyfes, les trois mues de 1975 et 1977 marquées au 1 avril ont eu lieu en réalité le $\mathbf{2 3}$ ou le 24 mars. Dans l'ensemble, nous avons cherché à illustrer différents types de cycles alimentaires, indépendamment de leur fréquence réelle. Toutefois, pour $V$. ammodyłes, la multiplication des exemples est destinée à montrer la tendance habituelle à une certaine similitude lorsque les conditions sont voisines. 
significatives. Il s'agit là essentiellement de la ration d'entretien chez de grands adultes dont la croissance pondérale était faible, mais cependant pas négligeable puisqu'elle atteignait en moyenne 4,39 $\pm 1,25$ p. 100 du poids du corps, par an. Comme pour le nombre des repas, il n'existe aucune corrélation entre la quantité relative de nourriture ingérée et la durée de la saison d'alimentation.

Périodes d'alimentation. - Les repas ne sont pas pris à intervalles réguliers et il existe, au cours de la saison d'alimentation, un certain nombre de périodes d'ingestion de nourriture, séparées par des périodes de jeûne. Lorsque la proie est de très grande taille relative, chacune de ces périodes d'alimentation peut d'ailleurs n'être représentée que par un seul repas. Le nombre et la date de ces périodes d'alimentation dépend de nombreux facteurs, notamment du cycle sexuel (les mâles ne se nourrissent pas à l'époque des accouplements) et du cycle des mues, qui diffèrent d'une espèce à l'autre.

Chez Vipera berus il existe habituellement trois périodes d'alimentation, la première au début de juin, la deuxième en juillet, la troisième à la fin d'août ou au début de septembre. On retrouve également trois périodes d'alimentation chez $V$. ammodytes, fin mai-début juin, en juillet et en août. Cette dernière période est brève et peut manquer.

Chez les autres espèces, on reconnaît généralement quatre périodes d'alimentation. Lorsqu'il y a deux mues par an, la première période d'alimentation se situe à la fin d'arril ou au début de mai, la deuxième en juin, la troisième en juillet ef la quatrième en août. La première et la dernière de ces périodes, ou bien l'une d'entre elles, manquent assez souvent. Lorsqu'il existe trois mues par an, ce qui est particulièrement fréquent chez $V$. aspis, les cycles alimentaires sont plus ou moins décalés et les deux ou trois premières périodes d'alimentation un peu plus précoces.

Mâles matures n'ayant pas terminé leur croissance. - Comme chez la plupart des Serpents, la maturité sexuelle des Vipères mâles intervient avant le moment de l'infléchissement rapide de la courbe de croissance. Le cycle sexuel et le cycle des mues de ces jeunes adultes sont identiques à ceux des grands mâles, mais la proportion de nourriture ingérée est beaucoup plus élevée : en moyenne 189 p. 100 du poids de l'animal. soit 66 p. 100 de plus que chez les grands adultes, la différence entre les deux groupes étant très significative $(p<0,001)$. Le rythme des périodes d'alimentation n'est généralement pas perturbé, mais aucune d'entre elles ne manque et les périodes de jeûnes qui les séparent son plus courtes. La figure 1 donne un exemple de ces différences chez V. aspis no 11, encore en pleine croissance en 1975, mais qui ne l'était plus en 1977.

Influence du climat. - L'année 1976, exceptionnellement chaude ( ${ }^{1}$ ), a constiłué une expérience naturelle intéressante et, de ce fait, les résultats obtenus cet été là méritent d'être interprétés à part. Lorsqu'elle se situaif avant la première mue, la date de la première période d'alimentation n'a pas été modifiée chez Vipera seoanei et $V$. aspis. Dans tous les autres cas, le premier repas eut lieu deux semaines plus tôt en moyenne, la saison d'alimentation étant raccourcie de 2 à 4 semaines chez toutes les

$\left({ }^{1}\right)$ Dans l'abri météorologique situé auprès des enclos, la température de l'air à $50 \mathrm{~cm}$ de hauteur a atteint ou dépassé $30^{\circ} \mathrm{C}$ au cours de 54 journées en 1976, contre 14,5 jours en moyenne pour les autres années entre 1972 eł 1977. 
espèces à l'exception de $V$. ammodyfes. Néanmoins, la proportion de nourriture ingérée a été significativement supérieure à la moyenne des autres années $(p<0,02)$, avec 142 p. 100 du poids de l'animal au lieu de 113 p. 100.

\section{Femelles}

Femelles dans la deuxième année d'un cycle triennal. - Les femelles dans la deuxième année d'un cycle triennal, c'est-à-dire celles qui ne sont ni reproductrices, ni postparturientes, se trouvent dans une situation assez comparable à celle des mâles, puisqu'elles n'ont guère à assurer que leur ration d'entretien. Si la durée de la saison d'alimentation est un peu plus longue (en moyenne 97 jours au lieu de 84), la ration alimentaire est très voisine de celle des grands mâles (respectivement 117 et 113 p. 100 du poids des animaux). On peut reconnaître trois périodes d'alimentation, la première, inconstante, fin mai-début juin, la deuxième fin juin-début juillet, la troisième en août. Ce rythme n'est guère modifié par l'existence de trois mues au lieu de deux.

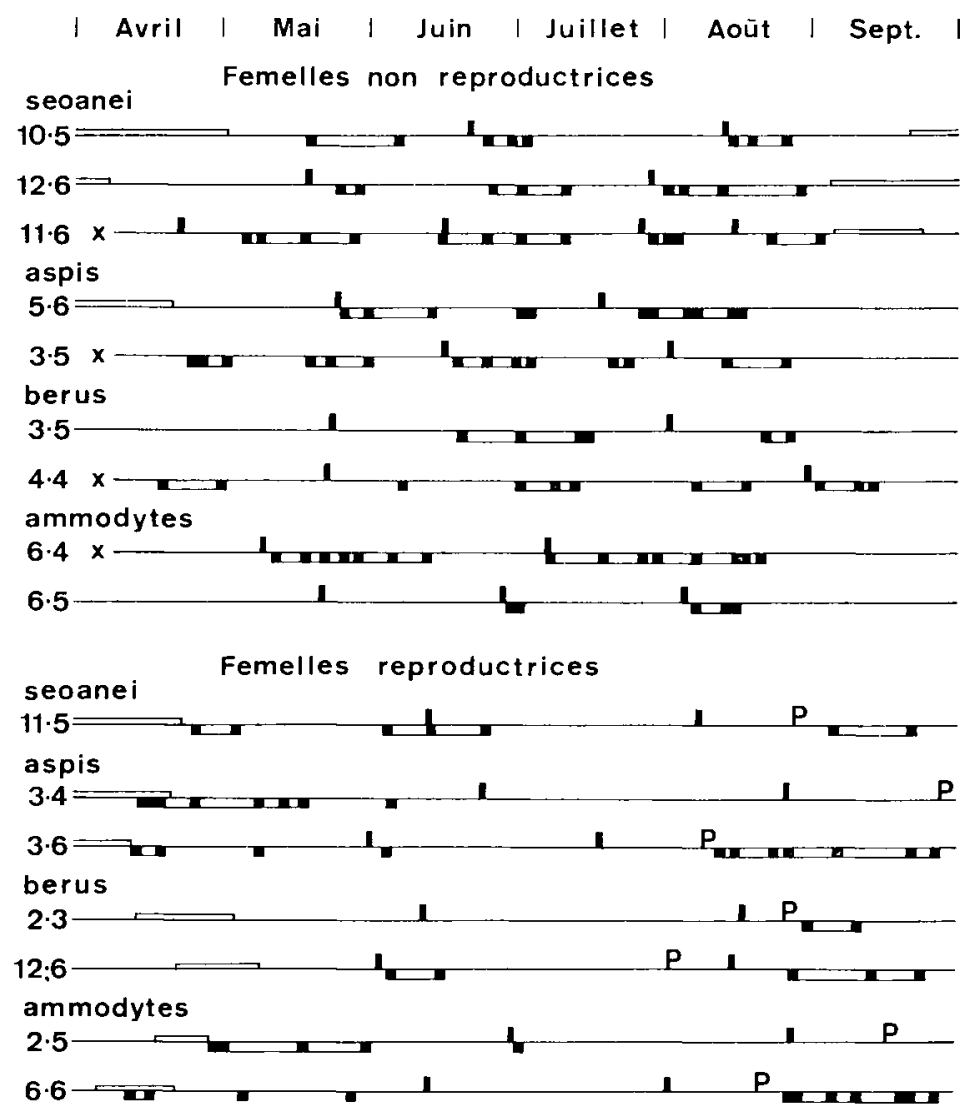

FHG. 2. - Cycles alimentaires, mues et périodes d'accouplement chez quelques Vipères femelles, à divers stades de leurs cycles sexuels (résultats individuels). $x=$ femelles postparturientes. $P=$ date de la parturition. Par ailleurs, même légende que fig. 1. 
Femelles reproductrices. - Le plus souvent, les femelles de V. berus ne s'alimentent qu'après la parturition, en septembre. Les deux individus pesés dans ces conditions ont, en deux repas chacun, ingéré respectivement 67,3 et 70,6 p. 100 de leur poids. Les femelles des autres espèces, au contraire, présentent toutes une saison d'alimentation vernale mais, en raison d'une parturition plus tardive, elles ne se nourrissent qu'exceptionnellement à l'automne. La saison d'alimentation vernale, d'une durée moyenne de 67 jours (tabl. 1) est constituée par deux ou peut-être trois périodes mal délimitées comprises entre la mi-avril ef le début de juillet. La proportion de nourriture ingérée dans ces conditions est faible : en moyenne 55 p. 100 du poids de la femelle pesée à l'automne précédent ou lors des premières sorties.

En 1976, chez V. seoanei, V. aspis et $V$. ammodytes (4 femelles), le premier repas a été plus précoce de deux semaines environ. La saison d'alimentation vernale a cependant été plus courte que d'ordinaire (18 à 53 jours, $m=47 \pm 9,9)$ ef la proportion de nourriture ingérée à ce moment encore plus faible $(35,6$ d̀ 37,5 p. 100 du poids de l'animal). Mais, la parturition étant très précoce (mi-août au lieu de septembre-octobre), il y eut une période d'alimentation automnale de 31 à 45 jours $(m=37 \pm 3,6)$, au cours de laquelle ces Vipères ont ingéré entre 97,8 et 123, 9 p. 100 de leur poids de nourriture $(m=113,1 \pm 6,8)$. Chez $V$. berus, les deux femelles étudiées ont mis bas le 3 août, avant la deuxième mue, mais elles n'ont commencé à s'alimenter qu'après cette mue, à la fin d'août. La durée de la période d'alimentation automnale a cependant été plus longue que les autres années, en moyenne 26 jours au lieu de 12, au cours desquels l'une des femelles a absorbé, en trois repas, 105,9 p. 100 de son poids de nourriture. Elle avait, en outre, pris deux repas en juin, ce qui est exceptionnel chez cette espèce.

Femelles postparturientes. - L'année suivant la reproduction, les femelles sortent de bonne heure au printemps et, au cours d'une longue saison d'alimentation, elles absorbent une quantité très élevée de nourriture (tabl. 1). II y a 4 ou plus souvent 5 périodes d'alimentation bien distinctes, entre la fin d'avril et la mi-septembre, sauf chez $V$. ammodytes où nous n'avons reconnu que deux longues périodes, suivant chacune des mues qui sont précoces.

En 1976, chez trois individus seulement de $V$. seoanei et $V$. aspis, nous n'avons pas constaté de différences significatives de la durée de la saison d'alimentation ou de la proportion de nourriture absorbée.

\section{Cycles alimentaires et rapports avec les mues}

Les périodes d'alimentation ne sont pas toujours faciles à différencier et dans quelques cas (par exemple fig. 1, V. ammodytes, mâle 1, 1976) l'opération serait purement arbitraire. En outre, puisqu'elles peuvent être constituées par un seul repas important, le calcul de leur durée n'a pas grande signification. On peut simplement constater que cette durée dépasse rarement trois semaines et que des périodes d'alimentation s'étendant apparemment sur plus d'un mois correspondent à des cas particuliers, notamment les femelles reproductrices à l'automne 1976.

Le premier repas d'une période d'alimentation a lieu le plus souvent dans les jours ou même les heures qui suivent la mue (fig. 3). Néanmoins, dans un certain nombre de cas la première proie n'est acceptée qu'une ou deux semaines après la mue. L'examen des données individuelles montre qu'un premier repas pris au cours 
des 4 ou 5 jours suivant la mue correspond soit à la reprise d'une période d'alimentation interrompue par la mue, soit, bien plus souvent, à une période d'alimentation dont le début coïncide avec la mue ou a été légèrement retardé par celle-ci. Les repas plus tardifs, surtout ceux qui n'ont lieu que plus de 10 jours après la mue, indiquent une période d'alimentation non synchronisée avec la mue.

Le laps de temps qui s'écoule entre le dernier repas et la mue (fig. 3) est très variable. Ce dernier repas correspond habituellement au début d'une période d'alimentation lorsqu'il précède la mue de 1 à 3 jours, à une période d'alimentation écourtée ou interrompue par la mue lorsqu'il la précède de 3 à 14 jours et à la fin d'une période d'alimentation lorsqu'il précède la mue de plus de 12 à 14 jours.

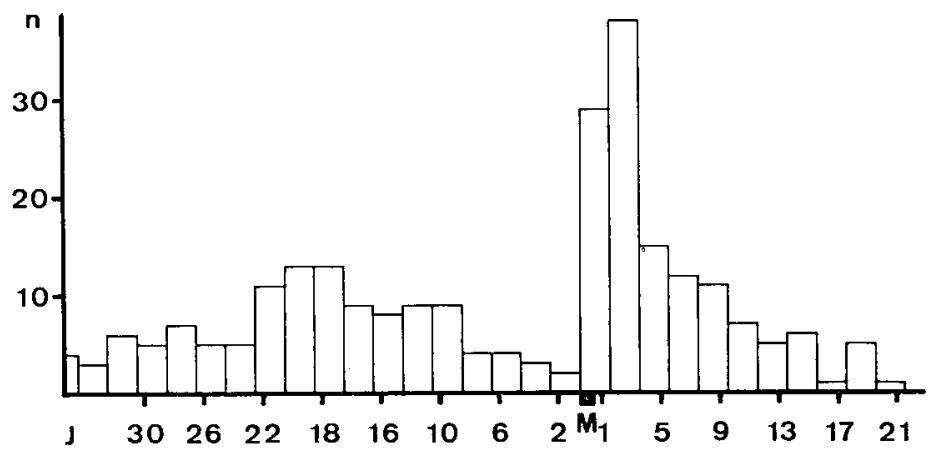

FIG. 3. - Temps séparant la mue du dernier repas qui la précède ef du premier repas qui la suif. En abcisses : temps en jours (J). En ordonnées : nombre d'observations ( $n$ ). $M=$ mue, prise comme point de départ. A gauche de la mue, les observations du dernier repas qui la précède. A droite de la mue, les observations du premier repas qui la suit.

\section{Discussion.}

Le cycle annuel d'alimentation des Vipères européennes était déjà connu dans ses grandes lignes grâce aux observations faites dans le milieu naturel (Rollinat, 1934 ; Saint-Girons, 1952 ; Viitanen, 1967 ; Prestt, 1971 ; Duguy, 1972). On constate que si la durée totale de la saison d'activité varie beaucoup selon les climats (elle peut être réduite à quatre mois et demi en montagne), les différences sont bien moindres en ce qui concerne la saison d'alimentation. C'est bien ce que nous montre la différence entre 1976 et les autres années. Par ailleurs, nos résultats confirment et précisent les observations antérieures sur les différences dues à l'âge et à l'état sexuel.

Les rations alimentaires annuelles des Vipères présentent, dans nos conditions semi-naturelles, d'importantes variations individuelles. II apparaît néanmoins, de façon très nette, que la ration d'entretien est faible, de l'ordre de 115 p. 100 du poids de l'animal, alors que la quantité de nourriture ingérée par an atteint en moyenne 190 p. 100 chez les jeunes mâles matures encore en cours de croissance et 276 p. 100 chez les femelles postparturientes. Au contraire les femelles gestantes, avec une ration alimentaire de 55 p. 100 de leur poids avant la vitellogenèse, vivent manifestement sur leurs réserves. Lors d'étés particulièrement chauds, les rations alimentaires fendent à augmenter chez la plupart des Vipères et, surtout, les parturitions étant plus 
précoces, les femelles peuvent reconstituer leurs réserves aussitôt après la mise bas, alors qu'elles ne le font d'ordinaire que l'année suivante.

Pomianowska $(1972,1974)$ a tenté d'établir un bilan énergétique chez Vipera berus. Les deux sexes confondus, elle obtient une dépense moyenne de $302 \mathrm{kcal}$ par an, qui serait couverte par l'ingestion d'environ $155 \mathrm{~g}$ de Rongeurs. D'après les données de cet auteur, nous avons calculé que la ration alimentaire annuelle de jeunes mâles matures encore en cours de crojssance correspondrait à 167 p. 100 de leur poids au moins et celle de femelles postparturientes à 212 p. 100, valeurs en bon accord avec nos mesures. Les résultats de Naulleau (1966) sur V. aspis concernent des individus maintenus constamment dans des cages chauffées pendant la journée et qui se nourrissent donc durant toute l'année. Dans ces conditions, la ration alimentaire annuelle de grands mâles a été en moyenne de 272 p. 100 de leur poids ( 212 à 310 p. 100) ce qui, compte tenu de la beaucoup plus longue durée de la saison d'alimentation, correspond à une ration mensuelle analogue à celle que nous avons trouvée.

Le fait que les Serpents se nourrissent beaucoup plus volontiers durant les jours qui suivent la mue et, au contraire, jeûnent plus ou moins durant les jours ou les semaines qui la précèdent, est connu depuis longtemps. Toutefois, à notre connaissance, le problème de la périodicité des cycles alimentaires à moyen terme n'a jamais fait l'objet d'une éfude systématique dans des conditions semi-naturelles. Même dans les conditions d'élevage habituelles, il n'existe guère que des données éparses, portanf sur peu d'individus (voir notamment Klauber, 1956, pour les Crotalinae). L'une d'entre elles est cependant particulièrement intéressante. En effet, Woodward (1933) a observé, chez une femelle isolée de la Vipère africaine Causus rhombeatus, un cycle alimentaire à peu près mensuel, rigoureusement lié au cycle des mues et au cycle reproductif. Le problème est manifestement plus complexe chez la majorité des Serpents, surtout dans les zones tempérées, ef la liaison entre les mues ef les cycles alimentaires moins rigide. En raison du nombre des paramètres impliqués, presque chaque animal représente, chaque année, un cas particulier. On reconnaît cependant, chez les Vipères européennes, des périodes d'alimentation distinctes dont le nombre et la date sont contrôlés par divers facteurs endogènes et exogènes (état des réserves lipidiques, cycle des mues, état sexuel, conditions météorologiques, etc...), mais qui n'en possèdent pas moins un rythme propre.

Il est certain que les caractéristiques des cycles alimentaires des Vipères jouent un rôle important dans la biologie des différentes espèces ef correspondent en partie à des adaptations écologiques. Pour ne citer qu'un exemple, on peut constater que les périodes d'alimentation des mâles ef celles des femelles reproductrices sont presque complémentaires, le mois de juin étant pratiquement la seule époque de pression de prédation commune de ces deux catégories.

Reçu en ooot 1978.

Accepté en septembre 1978.

\section{Références}

DUGUY R., 1963. Biologie de la latence hivernale chez Vipera aspis. Vie Milieu, 14, 311-444.

DUGUY R., 1972. Notes sur la biologie de Vipera aspis dans les Pyrénées. Terre Vie, 26, 98-117. KLAUBER L. M., 1956. Rattlesnakes. Vol.. 1, 708 pp. Univ. Calif. Press, Berkeley \& Los Angeles. 
NAULLEAU G., 1966. La biologie et le comportement prédateur de Vipera aspis au laboratoire et dans la nafure. 136 pp. P. Fanlac, Périgueux.

POMIANOWSKA I., 1972. Metabolic rate in adder (Vipera berus L.). Bull. Acad. pol. Sci., Sér. Sci. biol., 20, 143-146.

POMIANOWSKA-PILIPIUK I., 1974. Energy balance and food requirements of adult vipers Vipera berus (L.). Ekol. pol., 22, 195-211.

PRESTT I., 1971. An ecological study of the viper Vipera berus in southern Britain. J. Zool., London, 164, 373-418.

ROLLINAT R., 1934. La vie des reptiles de la France centrale. 343 pp. Delagrave, Paris.

SAINT-GIRONS H., 1952. Ecologie et éthologie des Vipères de France. Ann. Sci. nat., Zool., 11 e ser., 14, 263-341.

SAINT-GIRONS H., 1957. Le cycle sexuel chez Vipera aspis (L.), dans l'ovest de la France. Bull. biol. Fr. Belg., 151, 284-350.

SAINT-GIRONS H., 1976. Les différents types de cycles sexuels des mâles chez les Vipères européennes. C. R. Acad. Sci. Paris, Sér. D., 282, 1017-1019.

SAINT-GIRONS H., 1978. Thermorégulation comparée des Vipères d'Europe. Ełude biotélémétrique. Terre Vie, 32, 417-440.

VIITANEN P., 1967. Hibernation and seasonal movements of the viper, Vipera berus (L.), in southern Finland. Ann. Zool. fenn., 4, 472-548.

WOODWARD S. J., 1933. A few notes on the persistance of active spermatozoa in the african night adder, Causus rhombeatus. Proc. zool. Soc. London, 103, 189-190. 